\title{
PPAR y Agonist Pioglitazone in Combination With Cisplatinum Arrests a Chemotherapy-resistant Osteosarcoma PDOX Model
}

\author{
TAKASHI HIGUCHI ${ }^{1,2,3}$, JUN YAMAMOTO ${ }^{1,2}$, NORIHIKO SUGISAWA ${ }^{1,2}$, \\ YOSHIHIKO TASHIRO $^{1,2}$, HIROTO NISHINO ${ }^{1,2}$, NORIO YAMAMOTO $^{3}$, \\ KATSUHIRO HAYASHI ${ }^{3}$, HIROAKI KIMURA ${ }^{3}$, SHINJI MIWA ${ }^{3}$, KENTARO IGARASHI ${ }^{3}$, \\ MICHAEL BOUVET $^{2}$, SHREE RAM SINGH ${ }^{4}$, HIROYUKI TSUCHIYA ${ }^{3}$ and ROBERT M. HOFFMAN ${ }^{1,2}$ \\ ${ }^{1}$ AntiCancer, Inc., San Diego, CA, U.S.A.; \\ ${ }^{2}$ Department of Surgery, University of California, San Diego, CA, U.S.A.; \\ ${ }^{3}$ Department of Orthopedic Surgery, Kanazawa University, Kanazawa, Japan; \\ ${ }^{4}$ Basic Research Laboratory, Center for Cancer Research, National Cancer Institute, Frederick, MD, U.S.A.
}

\begin{abstract}
Background/Aim: Cisplatinum (CDDP) is a firstline drug in osteosarcoma treatment and the acquisition of resistance to CDDP is associated with a poor prognosis. Peroxisome proliferator-activated receptor gamma (PPAR $\gamma)$ is a nuclear hormone receptor that plays important roles in cell proliferation, differentiation, development, metabolism and cell death. Recently, PPAR $\gamma$ was reported to enhance the efficacy, overcome resistance, and decrease the toxicity of CDDP in various human cancers. In this study we tested whether pioglitazone (PIO), a PPAR $\gamma$ agonist, could overcome CDDP resistance in osteosarcoma. Materials and Methods: In this study, we used a human osteosarcoma cell line and a patient-derived orthotopic xenograft (PDOX) models of osteosarcoma. We measured cell viability of $143 B$ human osteosarcoma cells when treated with CDDP and PIO. We randomized PDOX models of osteosarcoma into four treatment groups: Group 1, Untreated control; Group 2, PIO alone; Group 3, CDDP alone; Group 4, a combination of $C D D P$ and PIO. Each group comprised six mice. Mice were treated for 14 days and tumor size and body weight were
\end{abstract}

This article is freely accessible online.

Correspondence to: Robert M. Hoffman, Ph.D., AntiCancer, Inc., 7917 Ostrow Street, San Diego, CA 92111, U.S.A. Tel: +18586542555, Fax: +18582684175, e-mail: all@anticancer.com; Shree Ram Singh, Ph.D., Basic Research Laboratory, Center for Cancer Research, National Cancer Institute, Frederick, MD 21702, U.S.A. Tel: +1 3018467331, e-mail: singhshr@mail.nih.gov; Hiroyuki Tsuchiya, Department of Orthopaedic Surgery, Kanazawa University, 13-1, Takara-machi, Kanazawa 920-8641, Japan. Tel: +810762652374, email: tsuchi@med.kanazawa-u.ac.jp

Key Words: Osteosarcoma, pioglitazone, cisplatinum, drug resistance, PDOX, PPAR $\gamma$. measured. Results: Cell viability of $143 B$ human osteosarcoma cells was significantly reduced when PIO (50 $\mu \mathrm{mol} / \mathrm{l})$ was combined with CDDP compared to CDDP alone. PDOX osteosarcoma tumors treated with the CDDP-PIO combination showed the strongest tumor growth inhibition compared to other treatment groups. PDOX osteosarcoma tumors treated with the CDDP-PIO combination had the least cancer cells and the most necrosis in histological section. Conclusion: These results suggest that combining PIO along with CDDP could be an effective treatment strategy for osteosarcoma and has important clinical potential for patients.

Cisplatinum (CDDP) is first-line therapy for osteosarcoma. However, its use is limited due to side-effects, such as nephrotoxicity, neuropathies, and/or bone marrow toxicity (1). Peroxisome proliferator-activated receptor gamma (PPAR $\gamma$ ) is a member of the nuclear hormone receptor superfamily. $\operatorname{PPAR} \gamma$ plays important roles in cell proliferation and differentiation, lipid metabolism, development, and cell death and is a candidate tumor suppressor gene (2). PPAR $\gamma$ ligands exert anti-tumor efficacy in various cancer models, however, monotherapy of PPAR $\gamma$ ligands showed a limited efficacy in clinical trials (3). Even though PPAR $\gamma$ ligands are less efficacious as monotherapy, in combination with traditional chemotherapy drugs, they showed improved clinical potential (3). We previously showed that the PPAR $\gamma$ ligand pioglitazone (PIO) could overcome doxorubicin-resistance in an osteosarcoma patient-derived orthotopic xenograft (PDOX) model (4). It has been demonstrated that PPAR $\gamma$ ligands combined with platinum-based drugs increase therapeutic efficacy in multiple cancer models such as nonsmall cell lung cancer (5), colon cancer (6), and ovarian cancer (6), but not in osteosarcoma. In the present study, we tested whether PIO could overcome CDDP-resistance in osteosarcoma PDOX model. 
A

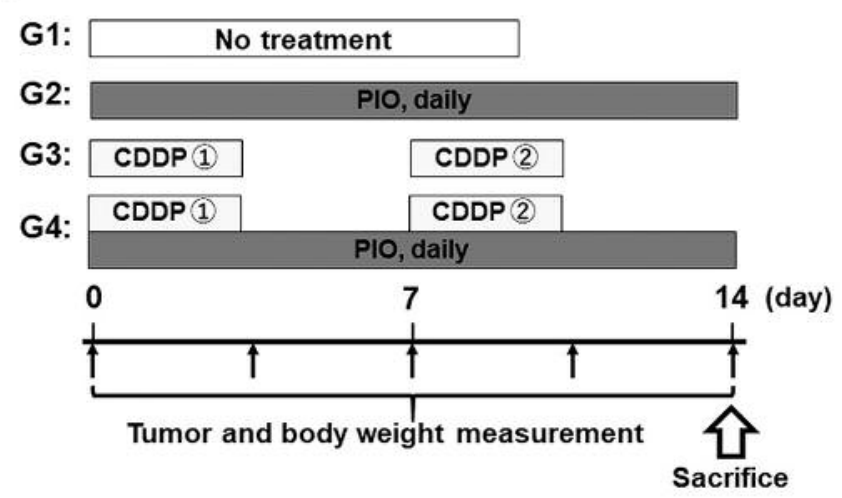

C

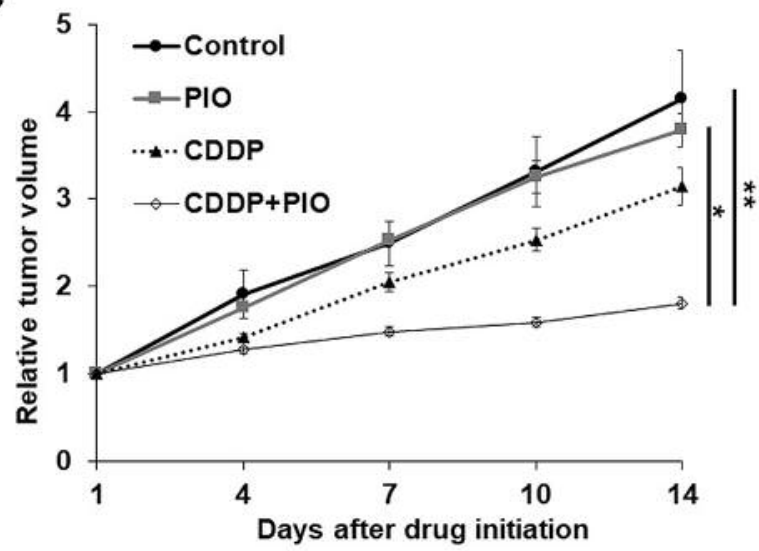

B

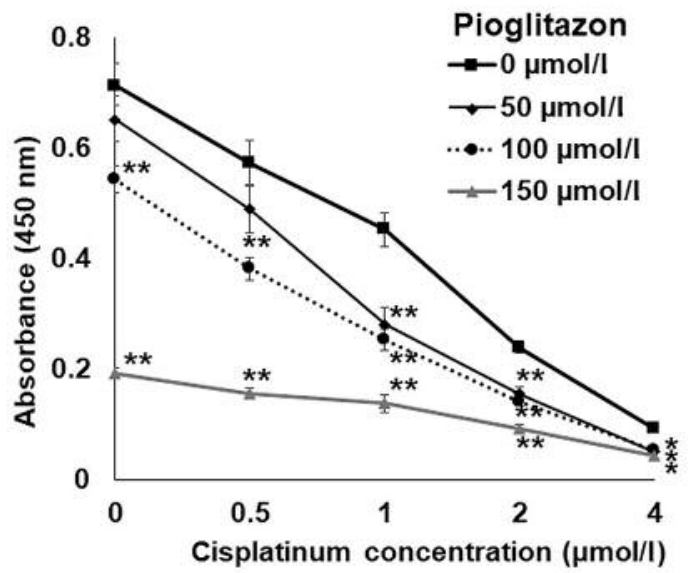

D

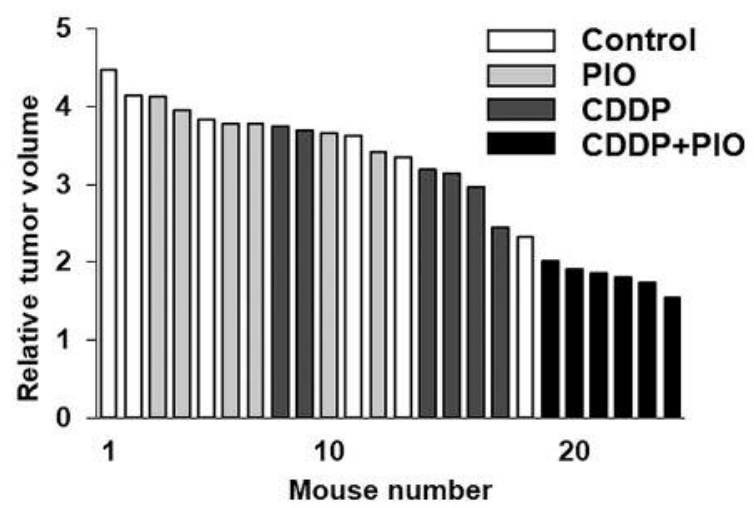

Figure 1. Treatment schema, cell viability of human osteosarcoma cells, and effect of drugs on the osteosarcoma-PDOX model. (A) Treatment protocol of the osteosarcoma-PDOX model. (B) Efficacy of CDDP and PIO on the viability of $143 B$ cells. *p<0.05; **p<0.01. (C) Effect of drugs on the tumor volume of the osteosarcoma-PDOX model. Line graphs show tumor volume at each time point after the onset of treatment relative to the initial tumor volume for each treatment and control group. (D) Waterfall plot show relative tumor volume at day 14 compared to the initial tumor volume for each mouse. $N=6$ mice/group. $* p<0.05 ; * *<<0.01$. Error bars: \pm SEM.

\section{Materials and Methods}

Cell line and cell culture. In the present study, we used the 143B human osteosarcoma cell line, which was maintained in RPMI-1640 (Mediatech, Inc. Manassas, VA, USA) with $10 \%$ fetal bovine serum (FBS) (4). Cell culture media used in this study were supplemented with penicillin and streptomycin to prevent bacterial contamination. Cells were cultured at $37^{\circ} \mathrm{C}$ with $95 \%$ air and $5 \%$ carbon dioxide $\left(\mathrm{CO}_{2}\right)$.

Cell viability assay. The cell proliferation assay was performed using a Cell Counting Kit-8 (CCK-8, Dojindo Laboratory, Mashikimachi, Japan). Detailed methods have been described in our previous publication (4). In brief, $143 \mathrm{~B}$ cells $\left(4 \times 10^{3}\right.$ cells/well) were seeded in 96-well plates. Cells were incubated for $24 \mathrm{hrs}$ at $37^{\circ} \mathrm{C}$. After that PIO $(0,50,100,150 \mathrm{umol} / \mathrm{l})$ and $\operatorname{CDDP}(0,5,1,2,4$ umol/l) were added to the medium and incubated for $48 \mathrm{~h}$. The medium was removed and the CCK- 8 reagent was added to the plates and incubated at $37^{\circ} \mathrm{C}$ for $2 \mathrm{~h}$. After the final incubation the absorbance was measured at $450 \mathrm{~nm}$ using a microplate reader
(iMark Microplate Absorbance Reader, Bio-Rad Laboratories, Inc., Hercules, CA, USA) (4).

Mice. In this study, we used athymic nu/nu nude mice (AntiCancer, Inc., San Diego, CA, USA). All mouse studies were performed using an AntiCancer, Inc. Institutional Animal Care and Use Committee (IACUC) protocol specifically approved for this study (4) and as per as the principles and procedures provided in the National Institutes of Health Guide for the Care and Use of Animals under Assurance Number A3873-1 $(4,7,8)$. Animal suffering was minimized by using anesthesia and analgesics during surgical procedures $(4,7,8)$.

Patient-derived tumor. In this study, we used a fresh biopsy sample from a 14-year-old boy with osteosarcoma located in the pelvis (4, $8)$. Detailed procedures for fragmentation of the samples and their subcutaneous implantation in nude mice were previously described $(4,8)$. The patient was not given any chemotherapy or radiotherapy before biopsy $(4,8)$. Written informed consent was obtained from the patient as part of a UCLA Institutional Review Board approved protocol (IRB\#10-001857) $(4,8)$. 

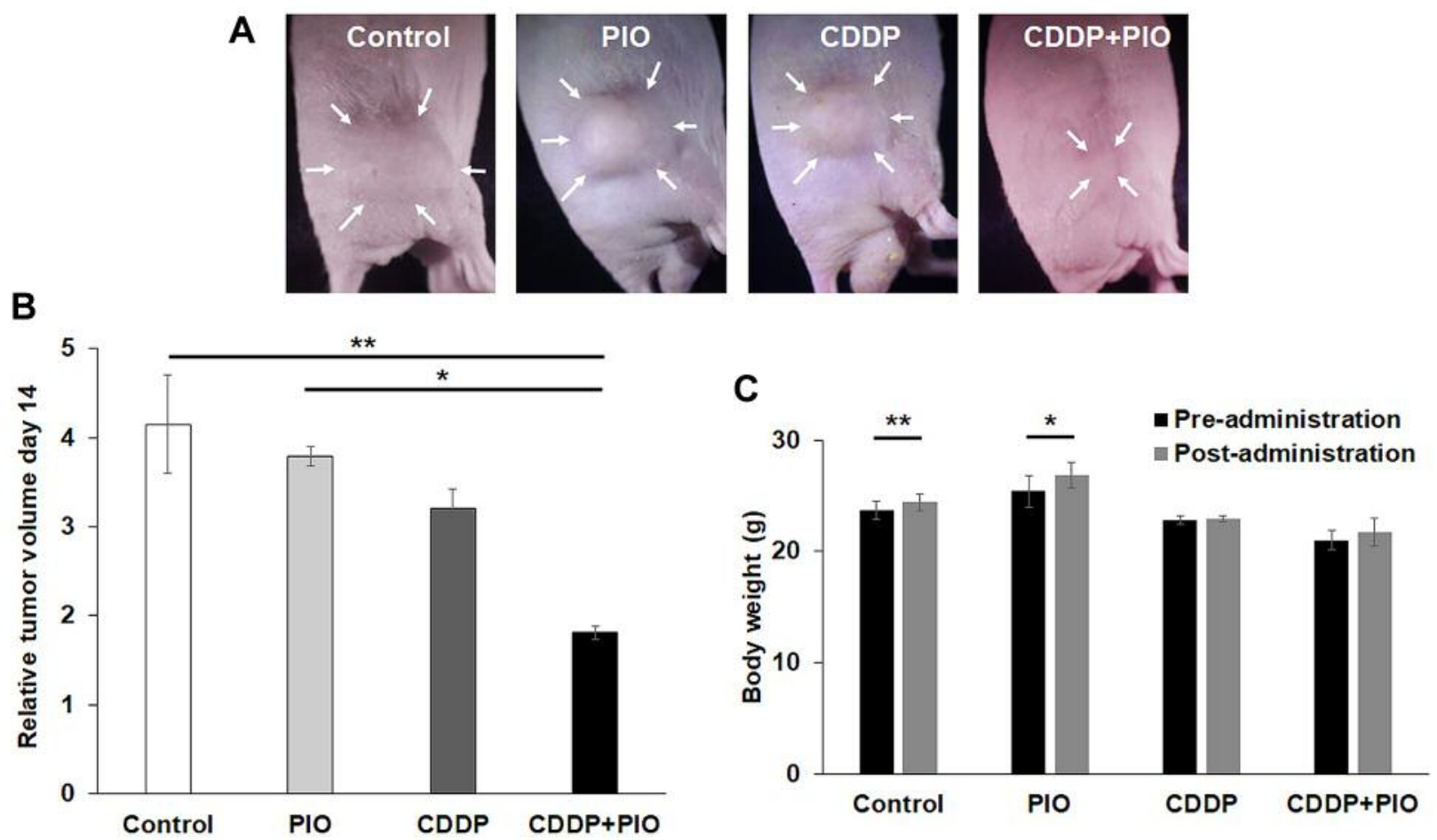

Figure 2. Tumor volume and mouse weight. (A) Representative photographs of osteosarcoma-PDOX mouse models from each treatment group on day 14. Arrows show the visible margin of the tumors. (B) Bar graphs show relative tumor volume of each treatment group on day 14 . N=6 mice/group. ${ }^{*} p<0.05 ;{ }^{*} p<0.01$. Error bars: \pm SEM. (C) Mouse body weight. Bar graphs show mouse body weight in each treatment or control group before and after treatment. $* p<0.05 ; * * p<0.01$. Error bars: \pm SEM.

Establishment of an osteosarcoma PDOX model. Surgical orthotopic implantation technique was used to establish an osteosarcoma PDOX model. Detailed procedures for mouse anesthesia, tumor fragmentation, orthotopic-implantation of tumors in the nude mouse distal femur and wound closures were described in our previous publications $(4,8)$.

Treatment regimen in the osteosarcoma PDOX model. We randomized PDOX models of osteosarcoma into four treatment groups. Each group contained 6 mice. They were treated for 2 weeks. The following four groups were established: (Figure 1A): G1, untreated control; G2, PIO (30 mg/kg, oral, daily) alone; G3, CDDP [6 mg/kg, intraperitoneal (i.p.) injection, once a week] alone; G4, CDDP (6 mg/kg, i.p., once a week) + PIO (30 mg/kg, oral, daily). Tumors were treated once they reached $100-200 \mathrm{~mm}^{3}$. Tumor length, width and mouse body weight were measured twice per week $(4,8)$. Tumor volume was calculated based on our previous publications $(4,8)$. Data are presented as mean \pm standard error of the mean (SEM).

Histological analysis. Fixation, paraffin sectioning and staining was performed as previously described $(4,8)$. Hematoxylin and eosin staining was performed according to a standard protocol. Cell proliferation was measured by immunohistochemical staining using an anti-Ki-67 antibody (Abcam Ltd., Cambridge, MA, USA), in combination with diamino-benzidine (DAB, Dako Japan Inc., Kyoto, Japan) staining, and hematoxylin counterstaining were performed according to manufacturer's protocols $(4,8)$.
Statistical analysis. Statistical analyses were performed using the EZR statistical software (Saitama Medical Center, Jichi Medical University). The Shapiro-Wilk test was used to assess the normal distribution. Homogeneity of variances across groups was verified using the Bartlett's test. One-way Analysis of Variance (ANOVA) with post hoc Tukey's HSD (Honestly Significant Difference) was used for the parametric test for comparing inter-groups. Parametric paired $t$-test was used to compare the means between two related groups. The Kruskal-Wallis with the Steel-Dwass non-parametric post hoc test was used for inter-group comparison. $p$-Values of less than 0.05 are regarded as statistically significant.

\section{Results}

Effect of CDDP and PIO on 143B osteosarcoma cells in vitro. $143 \mathrm{~B}$ cells treated with $\operatorname{CDDP}(0.5,1,2,4 \mu \mathrm{mol} /)$ for $48 \mathrm{~h}$ showed decreased viability in a dose-dependent manner (Figure 1B). When PIO (50, 100, $150 \mu \mathrm{mol} / \mathrm{l})$ was combined with CDDP, the viability of the $143 \mathrm{~B}$ cells was significantly decreased compared to the cells without PIO treatment, and in a dose-dependent manner (Figure 1B).

Effect of CDDP and PIO on the osteosarcoma-PDOX. We tested the osteosarcoma-PDOX tumors with the CDDP-PIO 

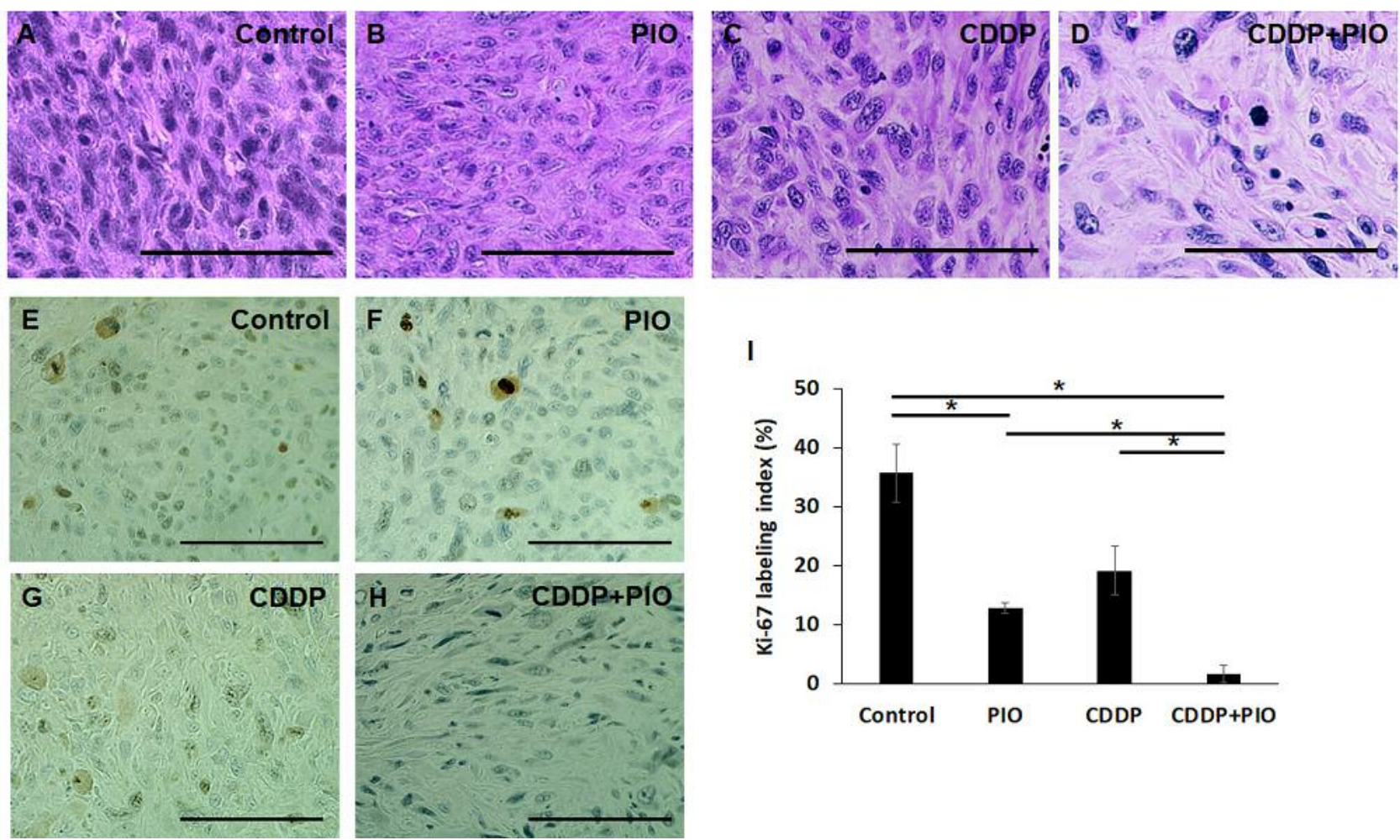

Figure 3. Tumor histology and cell proliferation. (A) Untreated control, (B) PIO alone, (C) CDDP alone, (D) CDDP + PIO. Scale bars: $100 \mu \mathrm{m}$. Ki-67 immunohistochemical staining $(E-H)$. (E) Control, $(F)$ PIO only, $(G) C D D P$ only, $(H) C D D P+P I O$. (I) Percentage of Ki-67 positive cells in control and each treatment group. Scale bars: $100 \mu m .{ }^{*} p<0.05$. Error bars: $\pm S E M$.

combination and found that this combination significantly inhibited the osteosarcoma PDOX tumor compared to the control $(p=0.002)$ and tumor treated with PIO alone $(p=0.02)$ (Figures 1C and D, 2A and B). However, no statistical difference was observed in osteosarcoma PDOX growth between the control and PIO-treated ( $p=0.90$ ) or CDDP-treated $(p=0.31)$ groups. A waterfall plot depict fold change in tumor volume for the individual tumors with each treatment at day 14 relative to the initial tumor volume (Figure 1D).

Effect of CDDP and PIO treatments on body weight. Final body weight of mice in the control and PIO alone groups significantly increased compared to the initial body weight ( $p=0.01$ and $p=0.03$, respectively). There was no significant difference in body weight among the other groups (Figure $2 \mathrm{C})$. There were no other observable side-effects or animal deaths in any group.

Tumor histology. Histological analysis showed that, the control tumors comprised viable highly-dense cancer cells with pleomorphic spindle-shaped cells. Osteosarcoma PDOX tumors treated with one dose of PIO or CDDP showed viable tumor cells with a lower cancer-cell density compared to the control. However, the lowest cancer-cell density was observed in osteosarcoma PDOX tumors treated with the combination of CDDP-PIO. In addition, we found that in this treatment group, many cancer cells were replaced by fibrous tissue that matched the anti-tumor efficacy of this combination in vivo (Figure 3A-D).

Immunohistochemical staining for proliferation marker (Ki67). Cell proliferation of cancer cells was evaluated after treatment on tumor sections using immunohistochemical staining with the Ki-67 proliferation marker. We found that osteosarcoma PDOX tumors treated with a combination of CDDP-PIO showed a significantly lower Ki-67 labeling index (mean Ki-67 labeling index, 1.67\%) compared to the control $(35.7 \%, p=0.01)$, CDDP alone $(19.2 \%, p=0.02)$, or PIO alone $(12.9 \%, p=0.02)$. We also found a statistical difference between PIO alone and the control $(p=0.04)$ (Figure 3E-I).

\section{Discussion}

Substantial evidence indicates that activating PPAR $\gamma$ synergistically enhances the efficacy of CDDP, minimizes its 
toxicity, and overcomes CDDP resistance $(3,5,6,9,10)$. Furthermore, a PPAR $\gamma$ ligand can decrease the incidence of nephrotoxicity, which is experienced in $30 \%$ of CDDPtreated patients, by reducing $\mathrm{TNF}-\alpha$, a crucial player in CDDP-induced nephrotoxicity $(11,12)$. PIO is a thiazolidinedione derivative and a synthetic PPAR $\gamma$ agonist, which is widely used as an anti-hyperglycemic agent $(2,13)$. In the present study, we found that PIO could inhibit proliferation of osteosarcoma cells in vitro, but not on the osteosarcoma PDOX model when it was administrated alone. However, tumor growth of the osteosarcoma PDOX model was significantly decreased when PIO was combined with CDDP, to which it was also not sensitive, indicating that PIO could enhance the efficacy of CDDP and overcome CDDPresistance.

We have established the PDOX mouse models of all cancer types $(4,14,15$, and references therein). We have also shown that the PDOX model is more patient-like compared to the subcutaneous patient-derived xenograft (PDX) model $(14,15)$. We also demonstrated that PDOX models maintain the original histological and molecular characteristics after transplantation in mice $(16,17)$. PDOX models provide osteosarcoma patients with specific and individualized treatment options (18-20). We previously showed that PIO could overcome doxorubicin resistance in the PDOX model of osteosarcoma (4).

In conclusion, the present study showed that the PDOX model is sufficiently powerful to precisely identify the effective-treatment options, such as the CDDP and PIO combination, for osteosarcoma. This result suggests that for the individual patient the osteosarcoma PDOX model could specifically distinguish both effective and ineffective drugs. For example, the tumor both in the patient and PDOX model is resistant to CDDP. The fact that the PPAR $\gamma$ agonist, PIO showed anti-proliferative activity on osteosarcoma cells in vitro and increase the efficacy of CDDP on the osteosarcoma PDOX model suggests potential clinical promise. Our results suggest the use of PDOX models for individual osteosarcoma patients (21).

\section{Conflicts of Interest}

AntiCancer Inc. uses PDOX models for contract research. TH, JY, NS, YT, HN, NY, KH, HK, SM, KI and RMH are or were unsalaried associates of AntiCancer Inc. There are no other competing financial interests.

\section{Authors' Contributions}

TH conceived and carried out the experiments with the collaboration of, JY, NS, YT, HN, NY, KH, HK, SM, and KI. TH, JY, NS, YT, HN, NY, KH, HK, SM, KI, MB, SRS, HT, and RMH were involved in data analysis and interpretation. RMH, SRS, and $\mathrm{TH}$ reviewed and revised the article.

\section{Acknowledgements}

This paper is dedicated to the memory of Reese Imhoff.

\section{References}

1 Janeway KA and Grier HE: Sequelae of osteosarcoma medical therapy: a review of rare acute toxicities and late effects. Lancet Oncol 11(7): 670-678, 2010. PMID: 20347613. DOI: 10.1016/S1470-2045(10)70062-0

2 Higuchi T, Takeuchi A, Munesue S, Yamamoto N, Hayashi K, Kimura H, Miwa S, Inatani H, Shimozaki S, Kato T, Aoki Y, Abe K, Taniguchi Y, Aiba H, Murakami H, Harashima A, Yamamoto Y and Tsuchiya $\mathrm{H}$ : Anti-tumor effects of a nonsteroidal antiinflammatory drug zaltoprofen on chondrosarcoma via activating peroxisome proliferator-activated receptor gamma and suppressing matrix metalloproteinase-2 expression. Cancer Med 7(5): 19441954, 2018. PMID: 29573200. DOI: 10.1002/cam4.1438

3 Skelhorne-Gross G and Nicol CJ: The key to unlocking the chemotherapeutic potential of PPARgamma ligands: having the right combination. PPAR Res 2012: 946943, 2012. PMID: 22966225. DOI: $10.1155 / 2012 / 946943$

4 Higuchi T, Sugisawa N, Miyake K, Oshiro H, Yamamoto N, Hayashi K, Kimura H, Miwa S, Igarashi K, Kline Z, Bouvet M, Singh SR, Tsuchiya H and Hoffman RM: Pioglitazone, an agonist of PPARgamma, reverses doxorubicin-resistance in an osteosarcoma patient-derived orthotopic xenograft model by downregulating P-glycoprotein expression. Biomed Pharmacother 118: 109356, 2019. PMID: 31545293. DOI: 10.1016/j.biopha. 2019.109356

5 Standiford TJ and Keshamouni VG: Chemotherapeutic drugs induce PPAR-gamma expression and show sequence-specific synergy with PPAR-gamma ligands in inhibition of non-small cell lung cancer. Neoplasia 10(6): 597-603, 2008. PMID: 18516296. DOI: $10.1593 /$ neo.08134

6 Girnun GD, Naseri E, Vafai SB, Qu L, Szwaya JD, Bronson R, Alberta JA and Spiegelman BM: Synergy between PPARgamma ligands and platinum-based drugs in cancer. Cancer Cell 11(5): 395-406, 2007. PMID: 17482130. DOI: 10.1016/j.ccr.2007.02.025

7 Miyake K, Kiyuna T, Kawaguchi K, Higuchi T, Oshiro H, Zhang Z, Wangsiricharoen S, Razmjooei S, Li Y, Nelson SD, Murakami T, Hiroshima Y, Matsuyama R, Bouvet M, Chawla SP, Singh SR, Endo I and Hoffman RM: Regorafenib regressed a doxorubicinresistant Ewing's sarcoma in a patient-derived orthotopic xenograft (PDOX) nude mouse model. Cancer Chemother Pharmacol 83(5): 809-815, 2019. PMID: 30758647. DOI: $10.1007 / \mathrm{s} 00280-019-03782-\mathrm{w}$

8 Higuchi T, Miyake K, Oshiro H, Sugisawa N, Yamamoto N, Hayashi K, Kimura H, Miwa S, Igarashi K, Chawla SP, Bouvet M, Singh SR, Tsuchiya H and Hoffman RM: Trabectedin and irinotecan combination regresses a cisplatinum-resistant osteosarcoma in a patient-derived orthotopic xenograft nudemouse model. Biochem Biophys Res Commun 513(2): 326-331, 2019. PMID: 30955860. DOI: 10.1016/j.bbrc.2019.03.191

9 Hamaguchi N, Hamada H, Miyoshi S, Irifune K, Ito R, Miyazaki $\mathrm{T}$ and Higaki J: In vitro and in vivo therapeutic efficacy of the PPAR-gamma agonist troglitazone in combination with cisplatin against malignant pleural mesothelioma cell growth. Cancer Sci 101(9): 1955-1964, 2010. PMID: 20608936. DOI: 10.1111/ j.1349-7006.2010.01632.x 
10 Quintao NLM, Santin JR, Stoeberl LC, Correa TP, Melato J and Costa R: Pharmacological treatment of chemotherapy-induced neuropathic pain: PPARgamma agonists as a promising Tool. Front Neurosci 13: 907, 2019. PMID: 31555078. DOI: 10.3389/ fnins.2019.00907

11 Zhang J, Zhang Y, Xiao F, Liu Y, Wang J, Gao H, Rong S, Yao $\mathrm{Y}, \mathrm{Li} \mathrm{J}$ and $\mathrm{Xu} \mathrm{G}$ : The peroxisome proliferator-activated receptor gamma agonist pioglitazone prevents NF-kappaB activation in cisplatin nephrotoxicity through the reduction of p65 acetylation via the AMPK-SIRT1/p300 pathway. Biochem Pharmacol 101: 100-111, 2016. PMID: 26673543. DOI: 10.1016/j.bcp. 2015.11.027

12 Mahmoud MF and El Shazly SM: Pioglitazone protects against cisplatin induced nephrotoxicity in rats and potentiates its anticancer activity against human renal adenocarcinoma cell lines. Food Chem Toxicol 51: 114-112, 2013. PMID: 22989705. DOI: $10.1016 /$ j.fct.2012.09.006

13 Vallee A, Vallee JN and Lecarpentier Y: PPARgamma agonists: potential treatment for autism spectrum disorder by inhibiting the canonical WNT/beta-catenin pathway. Mol Psychiatry 24(5): 643-652, 2019. PMID: 30104725. DOI: 10.1038/s41380-0180131-4

14 Igarashi K, Kawaguchi K, Kiyuna T, Murakami T, Miwa S, Nelson SD, Dry SM, Li Y, Singh A, Kimura H, Hayashi K, Yamamoto N, Tsuchiya H, Eilber FC and Hoffman RM: Patientderived orthotopic xenograft (PDOX) mouse model of adult rhabdomyosarcoma invades and recurs after resection in contrast to the subcutaneous ectopic model. Cell Cycle 16(1): 91-94, 2017. PMID: 27830986. DOI: 10.1080/15384101.2016.1252885

15 Hiroshima Y, Maawy A, Zhang Y, Zhang N, Murakami T, Chishima T, Tanaka K, Ichikawa Y, Bouvet M, Endo I and Hoffman RM: Patient-derived mouse models of cancer need to be orthotopic in order to evaluate targeted anti-metastatic therapy. Oncotarget 7(44): 71696-71702, 2016. PMID: 27765934. DOI: 10.18632 oncotarget.12322

16 Yamamoto M, Zhao M, Hiroshima Y, Zhang Y, Shurell E, Eilber FC, Bouvet M, Noda M and Hoffman RM: Efficacy of tumortargeting Salmonella A1-R on a melanoma patient-derived orthotopic xenograft (PDOX) nude-mouse model. PloS One 11(8): e0160882, 2016. PMID: 27500926. DOI: 10.1371/journal. pone. 0160882
17 Igarashi K, Kawaguchi K, Li S, Han Q, Tan Y, Murakami T, Kiyuna T, Miyake K, Miyake M, Singh AS, Eckardt MA, Nelson SD, Russell TA, Dry SM, Li Y, Yamamoto N, Hayashi K, Kimura H, Miwa S, Tsuchiya H, Singh SR, Eilber FC and Hoffman RM: Recombinant methioninase in combination with doxorubicin (DOX) overcomes first-line DOX resistance in a patient-derived orthotopic xenograft nude-mouse model of undifferentiated spindle-cell sarcoma. Cancer Lett 417: 168-173, 2018. PMID: 29306021. DOI: 10.1016/j.canlet.2017.12.028

18 Higuchi T, Sugisawa N, Miyake K, Oshiro H, Yamamoto N, Hayashi K, Kimura H, Miwa S, Igarashi K, Kline Z, Belt P, Chawla SP, Bouvet M, Singh SR, Tsuchiya H and Hoffman RM: Combination treatment with sorafenib and everolimus regresses a doxorubicin-resistant osteosarcoma in a PDOX mouse model. Anticancer Res 39(9): 4781-4786, 2019. PMID: 31519579. DOI: 10.21873/anticanres.13662

19 Kiyuna T, Tome Y, Miyake K, Murakami T, Oshiro H, Igarashi K, Kawaguchi K, Hsu J, Singh M, Li Y, Nelson S, Bouvet M, Singh SR, Kanaya F and Hoffman RM: Eribulin suppressed cisplatinum- and doxorubicin-resistant recurrent lung metastatic osteosarcoma in a patient-derived orthotopic xenograft mouse model. Anticancer Res 39(9): 4775-4779, 2019. PMID: 31519578. DOI: 10.21873/anticanres.13661

20 Higuchi T, Oshiro H, Miyake K, Sugisawa N, Han Q, Tan Y, Park J, Zhang Z, Razmjooei S, Yamamoto N, Hayashi K, Kimura H, Miwa S, Igarashi K, Bouvet M, Chawla SP, Singh SR, Tsuchiya $\mathrm{H}$ and Hoffman RM: Oral recombinant methioninase, combined with oral caffeine and injected cisplatinum, overcome cisplatinum-resistance and regresses patient-derived orthotopic xenograft model of osteosarcoma. Anticancer Res 39(9): 4653-4657, 2019. PMID: 31519563. DOI: 10.21873/anticanres.13646

21 Hoffman RM: The multiple uses of fluorescent proteins to visualize cancer in vivo. Nat Rev Cancer 5(10): 796-806, 2005. PMID: 16195751. DOI: 10.1038/nrc1717

Received October 16, 2019

Revised November 6, 2019 Accepted November 11, 2019 